\title{
Cluster-Driven Dynamical Arrest in Concentrated Lysozyme Solutions
}

\author{
Frédéric Cardinaux, ${ }^{\dagger}$ Emanuela Zaccarelli, ${ }^{*, \neq}$ Anna Stradner, ${ }^{*}{ }^{\top}$ Saskia Bucciarelli, ${ }^{\top}$ Bela Farago, ${ }^{\S}$ \\ Stefan U. Egelhaaf," Francesco Sciortino, ${ }^{\neq}$and Peter Schurtenberger ${ }^{\#}$ \\ ${ }^{\dagger}$ Department of Physics, University of Fribourg, Fribourg, Switzerland \\ ${ }^{\ddagger}$ Dipartimento di Fisica and CNR-ISC, Università di Roma La Sapienza, Roma, Italy \\ 'Adolphe Merkle Institute, University of Fribourg, Marly, Switzerland

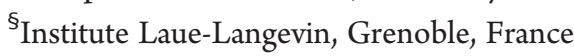 \\ "Condensed Matter Physics Laboratory, Heinrich-Heine University, Duesseldorf, Germany \\ \#Division of Physical Chemistry, Lund University, Lund, Sweden
}

\begin{abstract}
We present a detailed experimental and numerical study of the structural and dynamical properties of salt-free lysozyme solutions. In particular, by combining small-angle X-ray scattering (SAXS) data with neutron spin echo (NSE) and rheology experiments, we are able to identify that an arrest transition takes place at

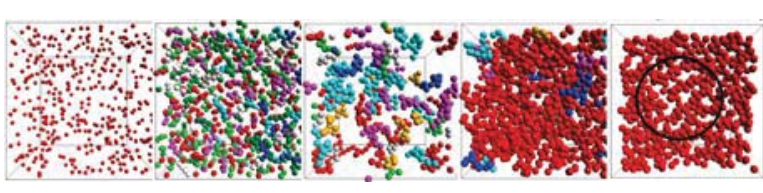
intermediate densities, driven by the slowing down of the cluster motion. Using an effective pair potential among proteins, based on the combination of short-range attraction and long-range repulsion, we account remarkably well for the peculiar volume fraction dependence of the effective structure factor measured by SAXS. We show that a transition from a monomer to a cluster-dominated fluid happens at volume fractions larger than $\phi \gtrsim 0.05$ where the close agreement between NSE measurements and Brownian dynamics simulations confirms the transient nature of the clusters. Clusters even stay transient above the geometric percolation found in simulation at $\phi>0.15$, though NSE reveals a cluster lifetime that becomes increasingly large and indicates a divergence of the diffusivity at $\phi \simeq 0.26$. Macroscopic measurements of the viscosity confirm this transition where the long-lived-nature of the clusters is at the origin of the simultaneous dynamical arrest at all length scales.
\end{abstract}

\section{INTRODUCTION}

The ability of colloidal particles to form equilibrium cluster phases under conditions where the particles interact via a potential consisting of a soft long-range repulsion and a shortrange attraction is well documented. ${ }^{1,2}$ In this class of systems, the short-range attraction drives cluster formation, whereas the increasing long-range repulsion due to the accumulating charge of the clusters limits their size. After an initial report ${ }^{3}$ that demonstrated the existence of such clusters in systems as diverse as concentrated lysozyme solutions at low ionic strength and weakly charged colloids in a low dielectric constant solvent with added nonadsorbing polymers (to induce a short-range attraction via a depletion mechanism), cluster phases have been demonstrated to exist in a large range of colloidal systems. ${ }^{4-8}$ In particular, the finding that equilibrium clusters may form in protein solutions under appropriate solution conditions has attracted considerable attention due to its potential biological significance in areas such as protein crystallization or protein condensation diseases. $^{9-16}$

The initial evidence of the existence of clusters in concentrated lysozyme solutions at low ionic strength has been obtained from the rather peculiar small-angle X-ray (SAXS) and small-angle neutron scattering (SANS) pattern obtained for these systems, ${ }^{3,17}$ where an analysis of the dependence of the scattering intensity as a function of the magnitude of the scattering vector $\mathbf{q}$ clearly reveals the existence of two peaks in the static structure factor $S(\mathbf{q})$. Surprisingly, both the low as well as the high-q peak were found to be almost independent of the volume fraction $\phi$ at sufficiently high values of $\phi$. This was subsequently interpreted as the signature of the presence of equilibrium clusters, where the low-q peak was attributed to a cluster-cluster interaction peak and thus denoted $\mathbf{q}_{\mathrm{c}}$, while the high-q peak was thought to reflect the monomermonomer correlations within the cluster and thus denoted $\mathbf{q}_{\mathrm{m}}$.

This interpretation was subsequently challenged by Shukla et al., ${ }^{18}$ who also conducted SANS and SAXS experiments with lysozyme solutions at low ionic strength. On the basis of the fact that they saw a clear shift of the cluster peak position at lower protein volume fractions, they questioned the existence of equilibrium clusters in lysozyme solutions. However, it has already been pointed out that for comparable experimental conditions, the claim of Shukla et al. relied in fact on scattering data that showed no significant differences to the earlier data on which the cluster model had been based. ${ }^{19,20}$ SAXS 
and SANS experiments on this system have now been performed by independent groups and show sets of data that are virtually identical, even though sometimes presented differently. $3,17-19,21,22$ Nevertheless, while there is now overwhelming evidence for the existence of clusters in lysozyme solutions at low ionic strength, there clearly remains the need for further experimental and theoretical investigations.

In particular, there is the need to obtain information on the finite lifetime of the equilibrium clusters. Initial attempts have been reported in a recent study of the dynamics of lysozyme clusters using neutron spin echo scattering experiments (NSE). ${ }^{21,22}$ The authors not only have shown evidence for the existence of equilibrium clusters, ${ }^{21}$ but they also found a virtually $\mathbf{q}$-independent dynamics for $\mathbf{q} \geq \mathbf{q}_{\mathrm{c}}$ indicating the absence of intracluster monomer dynamics. In this work, we now complement this information by presenting new, extensive results from a systematic study of the cluster dynamics on length scales $1 / \mathbf{q}_{\mathrm{c}}<L<1 / \mathbf{q}_{\mathrm{m}}$, where $L$ is the characteristic length over which we study diffusion. We therefore report NSE measurements over larger time and length scale intervals with respect to ref 21 under exactly the same conditions investigated in refs 3 and 18. These are combined with rheological measurements and Brownian dynamics (BD) computer simulations in an attempt to identify the signatures of the particles and of the clusters to the solution dynamics and to investigate the existence of a possible dynamical arrest or gelation.

Before presenting the results from our investigation of the dynamic properties of lysozyme clusters, we first summarize the static properties of lysozyme solutions at low ionic strength as obtained through a combination of SAXS, numerical simulations, and liquid state theory as they are essential for interpreting the NSE and rheology data. We then report neutron spin echo (NSE) measurements on the same sample in a wide range of lysozyme concentrations (volume fractions) at $T=5{ }^{\circ} \mathrm{C}$, providing evidence of dynamical arrest at $\phi \simeq$ 0.26 . At this density, clusters are the main building blocks of the structure of the solution, suggesting that dynamic arrest is driven by clusters, as opposed to single particles. These results are corroborated by additional viscosity measurements. We further test our potential model, which was developed to capture the structure of lysozyme solutions, ${ }^{23}$ by performing Brownian dynamics simulations in order to compare with the NSE data.

Our results show that for low $\phi$ the agreement for the dynamics appears to be quantitative. However, at high values of $\phi$, where cluster-cluster correlations become more and more important and eventually induce an arrest transition, the model starts to fail in describing the experimental data. Under these conditions, a decoupling of structure and dynamics seems to occur that makes it difficult to rely on theoretical tools such as mode coupling theory and simulations. We discuss this in terms of the pairwise additivity, which is most reliable at low volume fractions, and isotropy of the chosen model potential. We highlight the dominant role of the clusters, which appear to be much more stable in the real system than in simulations, underlining the important role of equilibrium clusters in the physics of salt-free lysozyme solutions at intermediate and large volume fractions.

\section{MATERIALS AND METHODS}

We used hen egg white lysozyme (Fluka-L7651, three times crystallized, dialyzed, and lyophilized) dispersed in 20 mM HEPES buffers ( $N$-(2-hydroxyethyl)piperazine- $N^{\prime}$-(2-ethanesulfonic acid, Sigma) in either water (Milli-Q, Millipore Corporation) or deuterium oxide (99.9\%, Cambridge Isotope Laboratories). We initially dissolved the lyophilized lysozyme at a concentration of $40 \mathrm{mg} / \mathrm{ml}$, stirred it at room temperature, and passed it through a $0.22 \mu \mathrm{m}$ filter to get rid of undissolved material. In order to remove any ionic impurities, this solution was thoroughly washed by replacing the solvent using an Amicon ultrafiltration stirring cell with a YM-10 membrane until the conductivity of the eluted solvent was comparable to that of the original buffer. The ultrafiltration stirring cell was further used to concentrate the protein solution. To ensure a constant $\mathrm{pH}$ during this process, the procedure was done in iterative steps by adding HEPES buffer at $\mathrm{pH} 7.8$ (adjusted with $\mathrm{NaOH}$ ) and checking the solution $\mathrm{pH}$ at each steps until the final, highly concentrated, stock solution had a $\mathrm{pH}$ of $7.8 \pm 0.1$. Under these $\mathrm{pH}$ conditions, lysozyme carries a net charge of $Z_{0}=+8$ electronic charges. Lower concentrations were prepared by diluting the stock solution with HEPES buffer at $\mathrm{pH}$ 7.8. The volume fraction was obtained from the protein concentrations $c$ measured by UV absorption spectroscopy at $280 \mathrm{~nm}$ (specific absorption coefficient $\left.E_{1 \mathrm{~cm}}^{1 \%}=26.4\right)$ and using $\phi=c / \rho$, where $\rho=1.351 \mathrm{~g} / \mathrm{cm}^{3}$ is the protein density. Note that the preparation steps are important in order to obtain consistent and reproducible results with the low salt conditions we are working under. Indeed, no finite amount of salt is added to the solution and the only contribution to the screening of the electrostatic repulsion is the buffer and the counterions. ${ }^{23}$ Ionic impurities or a shift of the solution $\mathrm{pH}$ during ultrafiltration may therefore lead to subsequent changes of the electrostatic repulsion.

SAXS scattering measurements were carried out with two pinhole cameras (NanoSTAR, Bruker AXS, and S-Max 3000 from Rigaku) equipped with a sealed tube $\left(\mathrm{Cu} \mathrm{K} \mathrm{K}_{\alpha}\right)$, a thermostatically regulated sample chamber, and a two-dimensional gas detector. The $\mathrm{q}$ range is $0.1-2 \mathrm{~nm}^{-1}$ for the NanoSTAR and $0.1-7 \mathrm{~nm}^{-1}$ for the S-Max 3000. SAXS measurements were performed to record the scattered intensity $I(\mathbf{q})$ of the sample as a function of the magnitude of the scattering vector $q=(4 \pi / \lambda) \sin (\theta / 2)$ with $\lambda$ being the wavelength of the radiation and $\theta$ as the scattering angle. $I(\mathbf{q})$ reflects both the shape of the particles as well as their spatial correlations, or structure. It is often expressed using an effective form factor $P_{\text {eff }}(\mathbf{q})$, where the shape of particles is averaged over all orientations, and an effective structure factor $S_{\text {eff }}(\mathbf{q})$. The scattering intensity then reads as $I(\mathbf{q})=c M K P_{\text {eff }}(\mathbf{q}) S_{\text {eff }}(\mathbf{q})$, where $c$ is the protein concentration, $M$ is its molar mass, and $K$ is a contrast term that depends on the physical properties of the scattering process. Practically, one extracts $P_{\text {eff }}$ from $I(\mathbf{q})$ at low concentration where no structure is expected in the solution (i.e., $S_{\text {eff }}(\mathbf{q}) \approx 1$ ), and $S_{\text {eff }}(\mathbf{q})$ is subsequently obtained for higher concentrations by division of $I(\mathbf{q})$ with $P_{\text {eff }}(\mathbf{q})$.

NSE measurements of the intermediate scattering function $f(\mathbf{q}, \tau)$ at length scales comparable with interparticle separation distance were performed at the neutron spin echo spectrometer IN-15 of the Institute Laue-Langevin (ILL) in Grenoble. ${ }^{24,25} \mathrm{We}$ combine measurements at two wavelengths, 9.2 and $14.5 \AA$, covering correlation times up to, respectively, 39 and $153 \mathrm{~ns}$, and wave vectors $\mathbf{q}$ ranging from 0.0097 to $0.235 \AA^{-1}$. Lysozyme solutions were contained in a 2 or $4 \mathrm{~mm}$ Hellma quartz cell and the temperature of $5{ }^{\circ} \mathrm{C}$ was controlled with a thermostatted sample holder on which dry nitrogen was constantly blown to prevent water condensation.

We performed molecular (MD) and $\mathrm{BD}$ simulations of a system composed of $N=2500$ particles of diameter $\sigma$ and mass $m$ in a cubic box of volume $V$, as a function of packing fraction 
$\phi=\pi \rho \sigma^{3} / 6$, where $\rho=N / V$ is the number density. We fixed $k_{\mathrm{B}} T=1$, while $\sigma$ and $\varepsilon$ are respectively the units of length and energy. Proteins were assumed to interact with a total potential $V_{\text {tot }}=V_{\mathrm{SR}}+V_{\mathrm{Y}}$, following, ${ }^{23}$ where $V_{\mathrm{SR}}$ models excluded-volume term plus the short-range attraction via a generalized LennardJones $2 \alpha-\alpha$ potential

$$
V_{\mathrm{SR}}(r)=4 \varepsilon\left[\left(\frac{\sigma}{r}\right)^{2 \alpha}-\left(\frac{\sigma}{r}\right)^{\alpha}\right]
$$

with $\alpha=90$, corresponding to an attractive range of approximately $3.6 \% \sigma$ (the latter parameter being fixed by comparison of the numerical static structure factors to the experimental ones). ${ }^{23}$ The long-range repulsion $V_{\mathrm{Y}}$ was modeled with a Yukawa potential, whose amplitude and screening length are fixed by the experimental conditions and follow the generalized onecomponent macroion model (GOCM) ${ }^{26}$

$$
V_{\mathrm{Y}}(r)=k_{\mathrm{B}} T L_{\mathrm{B}} Z_{0}^{2} X^{2} \frac{e^{-r / \xi}}{r}
$$

where $L_{\mathrm{B}}=e^{2} /\left(4 \pi \varepsilon_{0} \varepsilon k_{\mathrm{B}} T\right)$ is the Bjerrum length, $\xi=4 \pi L_{\mathrm{B}}\left(\rho Z_{0}+\right.$ $\left.2 \rho_{\mathrm{s}}\right)^{-1 / 2}$ is the Debye length, $\rho_{\mathrm{s}}$ is the salt number density in the buffer, and $X$ is a correction factor that depends on both $\phi$ and $\xi$ (eqs $11-15$ in ref 27). The resulting Yukawa potential gives a realistic description of the effective repulsion between proteins. Here the explicit $\phi$-dependence of $X$ incorporates the effect of screening of a protein by other proteins, whereas the contribution of additional counterions with increasing $\rho$ enters in the calculation of $\xi$. Under the chosen condition of low background electrolyte, we find it important to properly describe the change of $\xi$ and $X$ with $\phi$. Under excess salt, or infinite dilution, the GOCM reduces to the repulsive part of the classical DLVO potential. In ref 23, it was found that the total potential $V_{\text {tot }}$ has a constant depth $4.22 k_{\mathrm{B}} T$, arising from a balance of the attractive and repulsive components varying with $\phi$. The integration timestep was fixed to $\Delta t=5 \times 10^{-3}$ in units of $\left(m \sigma^{2} / \varepsilon\right)^{1 / 2}$. A cutoff at $r_{\mathrm{c}}=8 \xi$ was applied to reduce the computational effort without significantly altering the model because $V_{\text {tot }}\left(r_{\mathrm{c}}\right) / \varepsilon<10^{-3}$.

For the cluster analysis, proteins were considered to be bound when the separation between pairs of nearest-neighbor particles was smaller than $r_{\max }=1.065 \sigma$, a value that corresponds to the distance where $V_{\text {tot }}$ exhibits a maximum. Clusters were identified as groups of bonded proteins. To test for percolation, the simulation box was duplicated in all directions, and the ability of the largest cluster to span the replicated system was controlled. If the cluster in the simulation box did not connect with its copy in the duplicated system, then the configuration was assumed to be nonpercolating. The boundary between a percolating and a nonpercolating state point was then defined as the probability of observing infinite clusters in $50 \%$ of the configurations.

Viscosities were measured at shear rate ranging from 1 to $10^{3} \mathrm{~s}^{-1}$ with a stress controlled rheometer (MCR300 from PaarPhysica) using a cone and plate geometry and a solvent trap to minimize sample evaporation. For convenience, the rheology experiments were performed with samples in $\mathrm{H}_{2} \mathrm{O}$. A previous study demonstrated that the structure of the cluster phase is essentially unchanged when $\mathrm{H}_{2} \mathrm{O}$ is used instead of $\mathrm{D}_{2} \mathrm{O}$, although the onset of cluster formation occurs at slightly lower concentrations in $\mathrm{D}_{2} \mathrm{O}$ for a given temperature. ${ }^{17}$
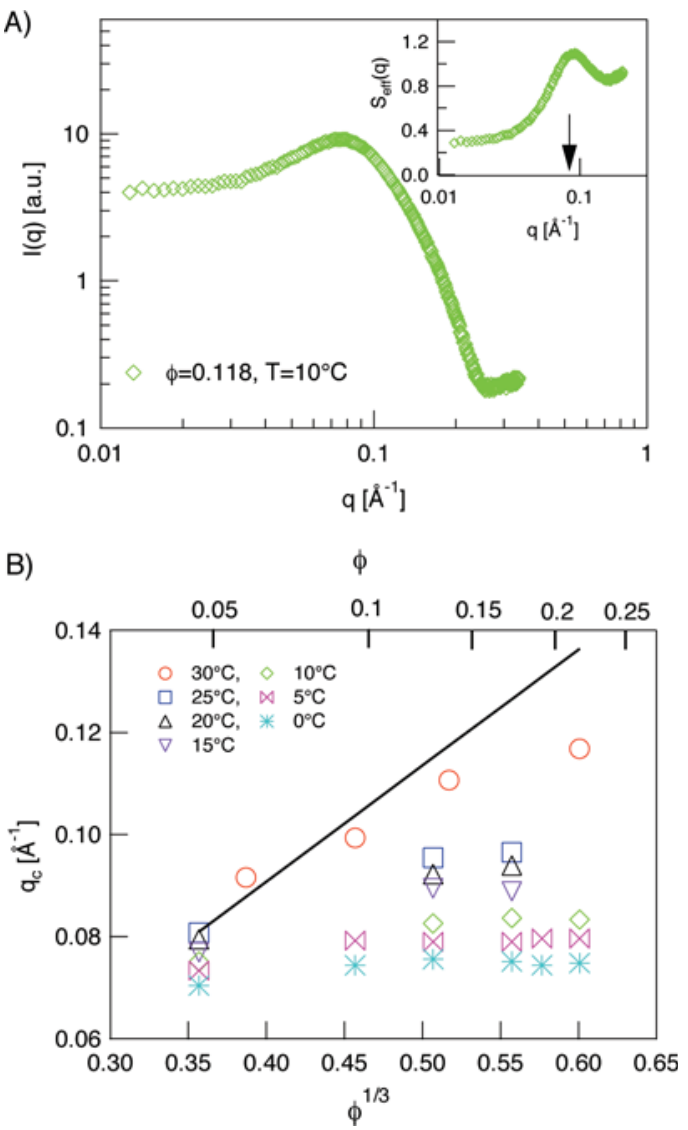

Figure 1. (A) Scattering intensity $I(\mathbf{q}) / \phi$ versus scattering vector $\mathbf{q}$ obtained with SAXS from a lysozyme solution at $T=10^{\circ} \mathrm{C}$ at a volume fraction $\phi=0.118$ in $\mathrm{H}_{2} \mathrm{O}$ and no added salt. Inset: Effective static structure factor $S_{\text {effi }}(\mathbf{q})$ obtained from the data shown in (A). (B) Concentration dependence of the position of the cluster peak $\mathbf{q}_{\mathrm{c}}$ at different temperatures. Also shown as a solid line is the expected behavior for purely repulsive charged particles interacting through a long-range screened Coulomb potential, where $\mathbf{q}_{\mathbf{c}} \sim n^{1 / 3}$ depends on the number density $n$ of particles only. Temperatures given are from top to bottom $30,25,20,15,10,5$, and $0{ }^{\circ} \mathrm{C}$. The nominal volume fractions can be read from the top axis.

\section{RESULTS}

Summary of Structural Properties of Lysozyme Solutions. As previous studies mostly lacked a systematic investigation of the static properties covering both low as well as high concentrations, we have now extended our earlier work on the characterization of the structural properties of lysozyme solutions using a combination of SAXS and computer simulations to much lower concentrations $0.003 \leq \phi$ for the simulations and $0.015 \leq \phi$ for the SAXS investigations. We believe that the results from this extension to low concentrations are essential in order to arrive at an understanding of the transition from a monomer-dominated molecular fluid to a cluster fluid.

Figure 1 illustrates the typical scattering intensity $I(\mathbf{q})$ and the resulting effective structure factor $S_{\text {eff }}(\mathbf{q})$ obtained for a lysozyme solution in a region of the phase diagram where equilibrium clusters are found. We have already published a detailed account of the volume fraction- and temperature dependence of the scattered intensity and the resulting effective structure factor $S_{\text {eff }}(\mathbf{q})$, and the bulk of the new SAXS results are thus given in the 


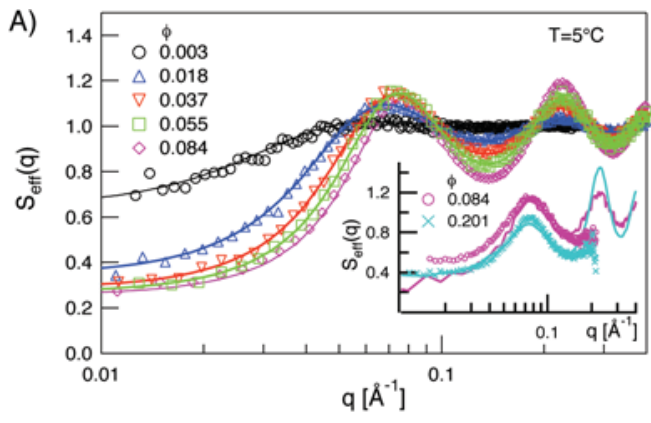

B)

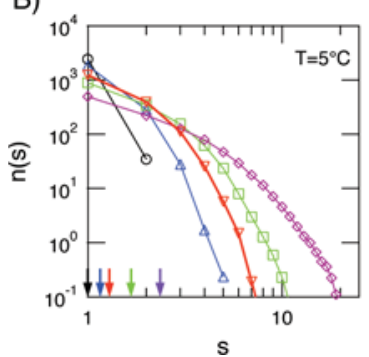

C)

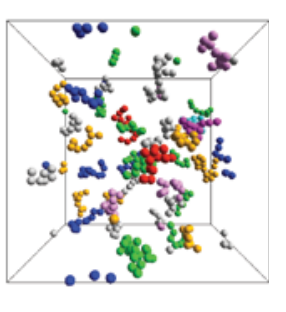

Figure 2. (A) Effective static structure factors for $T=5{ }^{\circ} \mathrm{C}$ obtained from PY theory (lines) and simulations (symbols) for the model of lysozyme solutions. Inset: Direct comparison of simulation results (lines) and experiments (symbols). (B) Cluster size distributions at $T=5{ }^{\circ} \mathrm{C}$ obtained from simulations for the same lysozyme $\phi$ as in (A). Also shown are average cluster aggregation numbers as arrows on the lower axis. (C) Snapshot from simulations at $\phi=0.084$, only clusters of size greater than 6 are shown for better visualization.

Supporting Information only. Figure 1B summarizes the volume fraction dependence of the position of the so-called cluster peak $\mathbf{q}$ at different temperatures ranging from $0{ }^{\circ} \mathrm{C} \leq T \leq 30^{\circ} \mathrm{C}$. As we will see later from the computer simulations, the cluster peak position provides important insight into the onset of cluster formation.

At the highest temperature, the SAXS data reveal a strong $\phi$-dependence of $\mathbf{q}_{\mathrm{c}}$ that follows the expected behavior of charged particles interacting via a weakly screened Coulomb repulsion, where the structure factor peak position is given by the number density $n \propto \phi$ of particles only and thus follows a power law of the form $\mathbf{q}_{\mathrm{c}} \sim \phi^{1 / 3}$. It is only at the highest volume fraction of $\phi=0.216$ that we see a clear deviation from this behavior beyond experimental uncertainty. At lower temperatures and in agreement with previously published results, ${ }^{17}$ short-range attractions between lysozyme becomes more dominant, and we observe that the peak position $\mathbf{q}_{\mathbf{c}}$ becomes almost independent of the protein volume fractions at high values of $\phi$. As we will see below from the computer simulations, this is a clear signature of a transition from a molecular fluid to a cluster fluid.

The existence of a peak in $S_{\text {eff }}(\mathbf{q})$ that occurs at $\mathbf{q}$-values below those predicted by $\mathbf{q}_{\mathrm{c}} \sim \phi^{1 / 3}$ does not yet allow to unambiguously conclude the existence of equilibrium clusters. ${ }^{22}$ We thus next concentrate on numerical simulations, where the starting point is our previously developed single-fit parameter potential composed of a short-range attraction and a long-range electrostatic repulsion. This mixed potential has shown to describe very well the static structure factors for intermediate and high volume fractions. ${ }^{23}$ From the simulations, we calculate the effective static structur factor $S_{\text {eff }}(\mathbf{q})$ as it can directly be compared with the experimentally determined $S_{\text {eff }}(\mathbf{q})$ obtained by dividing the

measured scattering intensity with the form factor of the lysozyme monomer. $S_{\text {eff }}(\mathbf{q})$ contains information on the correlations between monomers on relevant length scales, including information on the aggregates formed by the monomers and their spatial correlation. In contrast to other studies, ${ }^{22}$ we not only cover a large range of volume fractions, but also perform simulations under conditions corresponding to lower temperatures $\left(T=5{ }^{\circ} \mathrm{C}\right)$, where cluster formation has previously been shown to dominate. ${ }^{17,23}$ Figure 2 shows the corresponding data for a series of concentrations with $0.003 \leq \phi \leq 0.084$.

We find several key elements in $S_{\text {eff }}(\mathbf{q})$ that have previously been identified as characteristic for particles interacting via a mixed potential and a clear signature of cluster formation. First, a decreasing and rather low value of $S_{\text {eff }}(0)$ indicates that the system is dominated by repulsions at large length scales. At intermediate $\mathbf{q}$, a clear departure from what is expected for a purely repulsive system is observed, characterized by a cluster peak position $\mathbf{q}_{\mathrm{c}}$ that stays constant over a wide range of sufficiently high volume fractions. Finally, a high q peak corresponding to proteins at contact grows as the volume fraction increases. Previously, we already showed that a qualitative agreement between the effective structure factor from $\mathrm{MD}$ simulations and experiments is obtained for the intermediate and high q-peak positions as well as their amplitudes in the intermediate concentration range. ${ }^{23}$ This is again demonstrated in the inset of Figure 2A. Additionally, the low q-limit qualitatively mimics the observed decrease of $S_{\text {eff }}(0)$ with volume fraction, suggesting that the present potential also properly accounts for the compressibility $\left(\propto S(0)^{-1}\right)$ of the system. At this point, it is also important to point out that a constant cluster peak in $S(\mathbf{q})$ is not a specificity arising from the particular choice of parameters in our model but has been observed with mixed potentials under various conditions.

The simulations confirm the presence of equilibrium clusters for such an interaction potential and allow us to examine their equilibrium configurations. Figure $2 \mathrm{~B}$ shows the resulting distribution $n(s)$ of clusters of size $s$ for the same $\phi$ as in Figure 2A. For $\phi<0.055$, the solution is characterized by a size distribution that rapidly and monotonically decays to zero. The solution is essentially composed of monomers with a small amount of dimers and trimers. Above $\phi=0.055$, the size distribution broadens considerably and the fluids are composed of equilibrium clusters whose average size increases with $\phi$. For the current choice of parameters, the broadening of the size distribution at $\phi>0.055$ roughly coincides with the onset of the regime of constant $\mathbf{q}_{\mathrm{c}}$ for $\phi>0.08$. Figure $2 \mathrm{C}$ shows typical clusters obtained in the simulations at $\phi=0.084$. It is interesting to note that clusters exist in a quite broad distribution of sizes and shapes.

At larger $\phi$, the cluster size distribution progressively develops a power law behavior $n(s) \propto s^{-\tau}$ with an exponent $\tau \approx 2.2$ consistent with random percolation, and the system forms a space-spanning cluster for $\phi>0.148 .{ }^{23}$ The analysis of the simulations reveals that the clusters are highly transient, which renders the system still fluid, that is, on time scales larger than the average cluster lifetime it dynamically behaves like a fluid phase of clusters. Moreover, these clusters should not be confused with the transient clusters commonly observed when approaching a phase-separation boundary, whose spectral signature is a peak in $S(\mathbf{q})$ at $\mathbf{q} \rightarrow 0$.

We also include theoretical calculations for $S_{\text {eff }}(\mathbf{q})$ using integral equations theory (IE) within the Percus-Yevick (PY) approximation and additional simulations in the low volume 


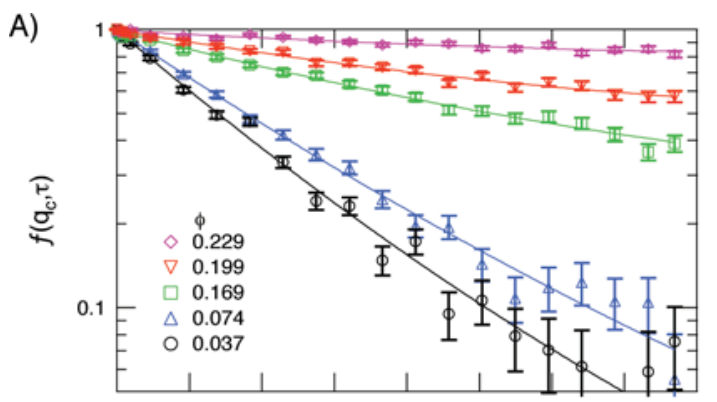

B)

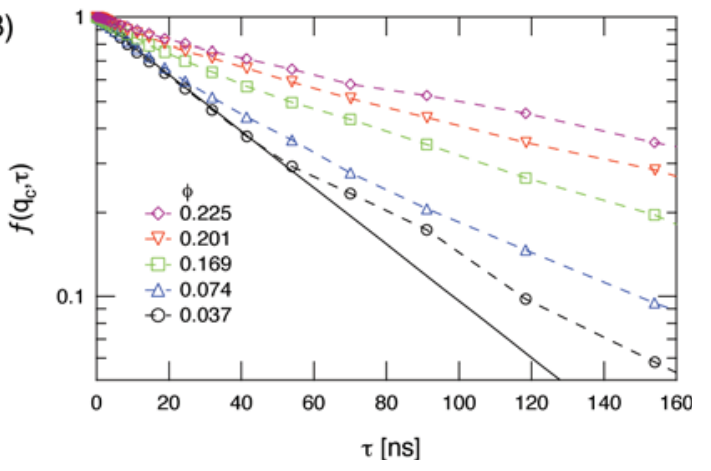

Figure 3. (A) Intermediate scattering function, $f(\mathbf{q}, \tau)$, measured with neutron spin echo at the cluster peak $\mathbf{q}=0.08 \AA^{-1}$ for lysozyme solutions at $5{ }^{\circ} \mathrm{C}$. Solid lines are cumulant fits to the data. (B) Corresponding data calculated from BD simulations. Time units are obtained by adjusting the $D_{0}$ value to that of the experimental system. The solid line is an exponential fit to the data at $\phi=0.037$.

fraction regime to illustrate the onset of equilibrium cluster formation. The results are shown in Figure 2A. PY calculations are essentially identical to the simulation results. It is interesting to note that this approach is equivalent to the fitting method used in ref 18 although we start with already known potential parameters (attraction range $\sim 3.6 \%$ of lysozyme diameter, attraction strength $\sim 4.22 k_{\mathrm{B}} T$, repulsion parameters fixed by experimental conditions; see ref 23 for more details). The remarkable agreement between experiments, numerical, and theoretical calculations suggests that a centrosymmetrical pair potential that includes short-range attraction and long-range electrostatic repulsion adequately describes the structure of this system.

Summarizing the investigation of the static properties, the simulations have provided insight into the mechanism leading to the formation of equilibrium clusters. Clusters result from the competition of a short-range attraction and a long-range electrostatic repulsion. The attraction among lysozyme particles favors the formation of clusters, while bulk aggregation is prevented by the long-range repulsion which increases with cluster size due to the increasing cluster charge and thus provides a stabilization mechanism. Simulations have also allowed us to characterize the onset of the cluster fluid, that is, the minimal amount of lysozyme in solutions required to form clusters, and indicated the existence of a static percolation threshold at $\phi>0.148$.

Short-Time Diffusion: Neutron Spin Echo Experiments and Brownian Dynamics Simulations. Having described the structural properties of the system, we now focus on the shorttime dynamical behavior of salt-free lysozyme solutions probed by NSE. ${ }^{24,25}$ In Figure 3A, we plot typical intermediate scattering functions (ISF) $f(\mathbf{q}, \tau)$ obtained by NSE for a set of volume fractions at a constant wave vector $\mathbf{q}_{\mathrm{c}}=0.08 \AA^{-1}$, corresponding

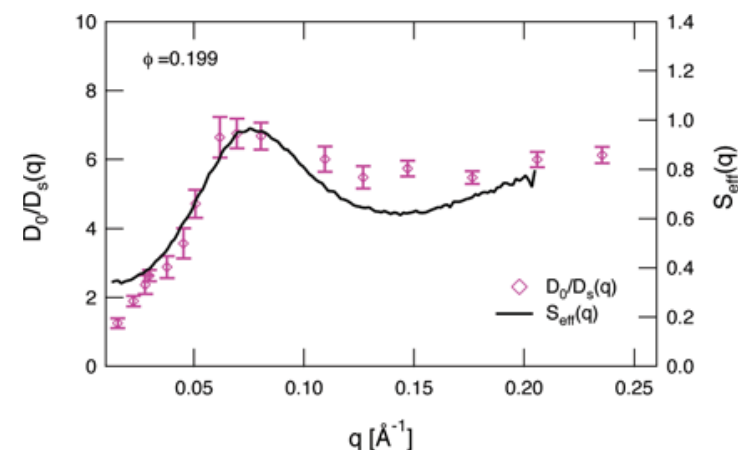

Figure 4. Normalized inverse short-time diffusion coefficient $D_{0} / D_{s}(\mathbf{q})$ and effective static structure factor $S_{\text {eff }}(\mathbf{q})$, extracted from fits of the ISF as described in the text, versus $\mathbf{q}$ for a volume fraction $\phi=0.199$.

to the cluster peak of $S_{\text {eff }}(\mathbf{q})$. At the lowest volume fractions, the initial decay rate of $f(\mathbf{q}, \tau)$ is reasonably well described by a single exponential. However, departure from a single exponential is clearly visible at higher values of $\tau$ for both experiments and simulations. We found the best fit to the data using a cumulant analysis, where the average relaxation rate of $f(\mathbf{q}, \tau)$ is obtained through the first cumulant $\Gamma(\mathbf{q})=-\lim _{\tau \rightarrow 0}[\mathrm{~d} \ln f(\mathbf{q}, \tau) / \mathrm{d} \tau]$, and the relative deviation from an exponential obtained using $Q=\mu / \Gamma^{2}$, where $\mu=\lim _{\tau \rightarrow 0}\left[\mathrm{~d}^{2} \ln f(\mathbf{q}, \tau) / \mathrm{d} \tau^{2}\right]$ is the second cumulant. ${ }^{28}$ We systematically checked that results for $\Gamma(\mathbf{q})$ are constant within the accuracy of the fits when the range of points considered is reduced to shorter correlation times $\tau$.

It is instructive to compare the time window offered by NSE with time scales at which dynamical processes occur in the system. First, particle velocities have completely relaxed on time scales of the smallest spin echo time, and collisions with solvent molecules effectively lead to a diffusive motion of particles. At this time scale, indirect interactions mediated by the solvent are also well established. Direct interactions become important at larger time scales where particles start to feel the structure of their surrounding. This typically occurs for $\tau>\tau_{\mathrm{I}}$, where $\tau_{\mathrm{I}}=d^{2} / D_{0}$ is the time required for a particle to freely diffuse over a structural distance $d$. Using the structure peak at contact for $d=2 \pi / \mathrm{q}_{\mathrm{m}}$ and $D_{0}=5.216 \times 10^{-11} \mathrm{~m}^{2} \mathrm{~s}^{-1}$ for the free diffusion coefficient of lysozyme in $\mathrm{D}_{2} \mathrm{O}$ at $5{ }^{\circ} \mathrm{C}$, we obtain an interaction time of $\tau_{I}=150 \mathrm{~ns}$, at the upper limit of our accessible time window. A similar analysis considering cluster diffusion would essentially shift $\tau_{\text {I }}$ to much larger times. This implies that NSE essentially probes the short time limit of the diffusion coefficient which is obtained from the first cumulant using $D_{s}(\mathbf{q})=\Gamma(\mathbf{q}) / \mathbf{q}^{2} \cdot{ }^{29}$

We now compare these results to those of Brownian dynamics simulations performed using the previously discussed effective lysozyme-lysozyme potential. It is important to point out that this potential has been devised in order to correctly describe the static structure of the system, but there is no a priori guarantee that it is also able to capture the dynamical behavior of the solutions. Hence, the comparison with experiments will provide a stringent test for this type of simplified effective interactions, which are most commonly used to describe experimental data. ${ }^{18,23,30}$

The ISF calculated from BD simulations are reported in Figure 3B for similar values of $\phi$ as studied experimentally at the cluster peak scattering vector. We find that the exponential behavior approximately holds in the range $0.5-50 \mathrm{~ns}$, that is, a 

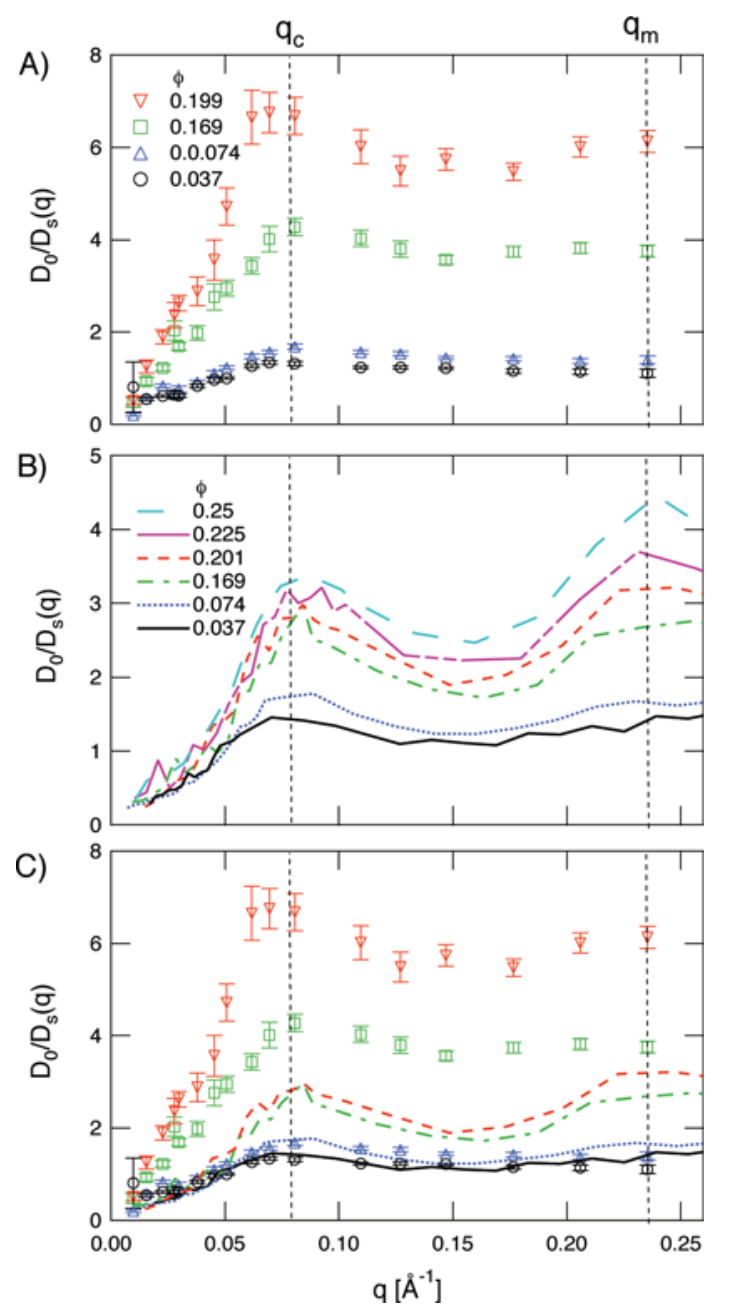

Figure 5. Inverse short-time diffusion coefficient $D_{0} / D_{s}(\mathbf{q})$ versus $\mathbf{q}$ for various studied $\phi$ : (A) experimental results, (B) simulation results, and (C) direct comparison of experiments and simulations. The vertical lines mark the location of the cluster peak $\mathbf{q}_{\mathrm{c}}$ and monomer peak $\mathbf{q}_{\mathrm{m}}$ observed in the static structure factors.

comparable range to experimental data. At larger times, deviations from a single exponential decay are also found. For consistency, the cumulant analysis is performed also for the simulation data. It can be immediately noticed from Figure 3 that, while for low $\phi$ the two sets of data are very similar, at larger volume fractions the experimental data show a much slower relaxation rate with respect to the $\mathrm{BD}$ simulations.

The q-dependence of the NSE results for the inverse shorttime diffusion coefficient $D_{0} / D_{s}(\mathbf{q})$ are shown for $\phi=0.199$ in Figure 4 after having normalized $D_{s}$ by its value at infinite dilution, $D_{0}$. A marked oscillation of $D_{0} / D_{s}(\mathbf{q})$ is observed at a wave vector corresponding to the cluster-cluster peak $\mathbf{q}_{c}$, as well as a less pronounced upturn at the monomer-monomer peak $\mathbf{q}_{\mathrm{m}}$. For purely repulsive colloidal systems and protein solutions, it is well established that the short-time diffusion coefficient can be expressed as $D_{0} / D_{s}(\mathbf{q})=S(\mathbf{q}) / H(\mathbf{q})$, where $H(\mathbf{q})$ is the hydrodynamic function. ${ }^{31,32}$ This proportionality of $D_{s}(\mathbf{q})^{-1}$ with $S(\mathbf{q})$ qualitatively holds also in our case, where particles interact with a mixed potential and form equilibrium clusters. However, it is important to point out that while the NSE data closely follow $S_{\text {eff }}(\mathbf{q})$ around the cluster peak, at higher $\mathbf{q}$-values the $\mathbf{q}$-dependence is much less pronounced. This indicates that at higher concentrations the dynamics on length scales smaller than the cluster size are dominated by the cluster diffusion and that monomers remain bound to the clusters on the time scale probed by the NSE experiments.

The data for all investigated volume fractions are summarized and compared with the results from the BD simulations in Figure 5. Let us first discuss the experimental results (Figure 5A). As already demonstrated in Figure 4 for $\phi=0.199$, we find for all volume fractions that the wave vector dependence of the dynamics remarkably mimics features observed in the static structure factor $S_{\text {eff }}(q){ }^{23}$ However, we also find for all these volume fractions that for $\mathbf{q} \gtrsim \mathbf{q}_{\mathrm{c}}$ the dynamics becomes almost independent of $\mathbf{q}$.

Although the same general features are observed for the simulation results in Figure 5B, this figure also shows significant differences when compared to the experimental data. While the presence of two distinct peaks, respectively at $\mathbf{q}_{\mathrm{c}}$ and $\mathbf{q}_{\mathrm{m}}$, is found in agreement with experiments, the oscillation in $D_{0} / D_{s}(\mathbf{q})$ at $\mathbf{q} \sim \mathbf{q}_{\mathrm{m}}$ corresponding to the dynamics on length scales comparable to the nearest-neighbor distance appears to be much more pronounced than for the experimental data, while the oscillation associated with the cluster peak increases less with packing fraction. However, for small values of $\phi$, the agreement between experimental and numerical results is almost quantitative, as evident from the direct comparison of data reported in Figure 5C. The use of Brownian dynamics (for example with respect to Newtonian dynamics) in the investigated short-time regime is crucial to these results, and also suggests that hydrodynamic effects, which have been neglected in our study, do not play a major role at these low densities. On the other hand, it is known that many-body hydrodynamic interactions will have effects in denser suspensions. ${ }^{33,34}$

We now comment on the meaning of the absolute values of the measured short-time diffusion coefficients. Our experiments in fact suggest that clusters do survive on time scales of our measurement window and effectively contribute to $f(\mathbf{q}, \tau)$. The relevant time scales can be estimated considering the time needed by two particles to escape their potential well. On the basis of the potential parameters of our model, we can estimate the escape time $\tau_{\mathrm{e}}$ assuming a simplified square-well shape for the attraction, retaining width $\Delta \sim 0.04 \sigma$ and depth $U \sim-5 k_{\mathrm{B}} T$. We obtain $\tau_{\mathrm{e}}=\left(\Delta^{2} / D_{0}\right) \exp \left(-U / k_{\mathrm{B}} T\right) \approx 45 \mathrm{~ns}$. This is of course a very rough estimate which, however, provides a lower boundary for $\tau_{\mathrm{e}}$ (since only dimers are considered) and clearly shows that clusters can persist within the time window offered by NSE. Therefore, the measured intermediate scattering functions do not only contain information on single particle diffusion but also on cluster diffusion. Given a sufficiently large cluster lifetime, we do in fact expect that the measured diffusion in the NSE time window is dominated by cluster motion even for $\mathbf{q}$-values that correspond to length scales of individual proteins around $\mathbf{q}_{\mathrm{m}}$.

The NSE experiments clearly provide evidence of a marked decrease of the short-time diffusion coefficient with increasing $\phi$. In particular it is found that the density fluctuations at length scales of the cluster-cluster and monomer-monomer separation distances are almost identical and follow the same $\phi$-dependence, as shown in Figure 6A. The data at fixed $q$ are well described by a power law fit of the form, $D_{0} / D_{\mathrm{s}} \sim\left(1-\phi / \phi_{\mathrm{g}}\right)^{-\alpha}$ with $\phi_{\mathrm{g}}=0.25$ and $\alpha \approx 1.25$. This power law behavior resembles that of systems approaching an ideal glass transition according to mode coupling theory (MCT), ${ }^{35}$ although the exponent found here is rather small compared to MCT predictions, probably due to the limited number of 

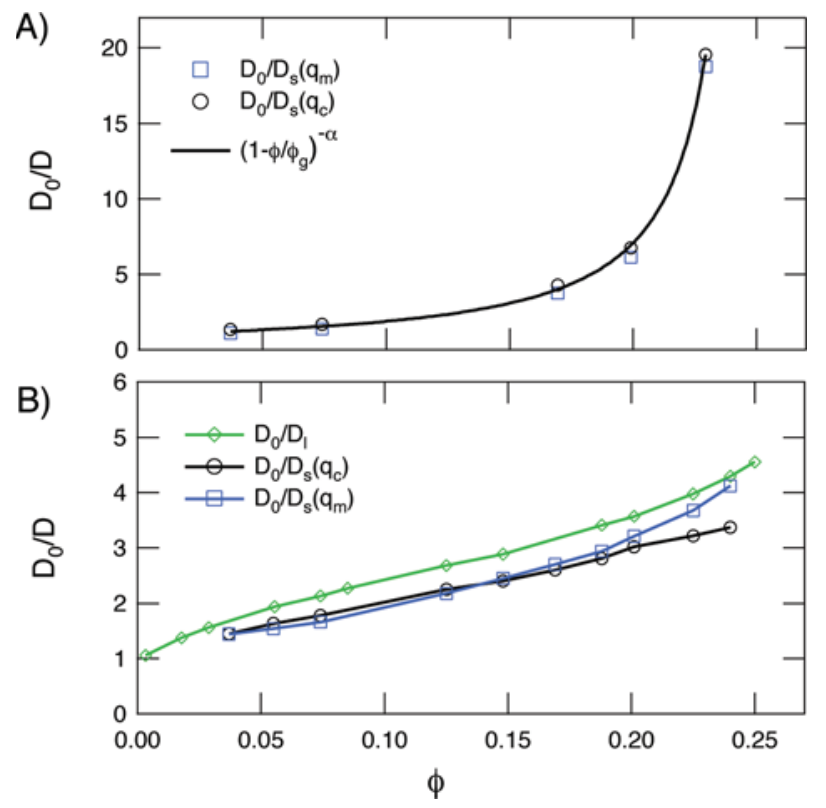

Figure 6. (A) Volume fraction dependence of the normalized reduced short- time diffusion coefficient $D_{0} / D_{\mathrm{s}}(\mathbf{q})$ at $\mathbf{q}_{\mathrm{c}}$ and $\mathbf{q}_{\mathrm{m}}$. Solid line is a fit using a glass-like divergence of the form $D_{0} / D_{\mathrm{s}} \sim\left(1-\phi / \phi_{\mathrm{g}}\right)^{-\alpha}$ with $\phi_{\mathrm{g}} \approx 0.25$ and $\alpha \approx 1.25$. (B) Same data from $\mathrm{BD}$ simulations. Also shown is the inverse long-time self-diffusion coefficient calculated from MSD.

measurements available as well as their relative distance from the arrest transition. Nevertheless, the fit provides an estimate of the volume fraction of arrest, $\phi_{\mathrm{g}} \approx 0.25$, at which the short-time diffusion tends to zero simultaneously for both the cluster-cluster and the monomer-monomer peak. Although such a fitting procedure provides only a very crude estimate of the arrest volume fraction, given the small number of measured data points and their large relative distance from $\phi_{g}$, our results suggest that lysozyme solutions undergo a cluster-driven dynamical arrest at $\phi_{\mathrm{g}}$. It is important to stress that, although we are dealing here with shorttime dynamics only, we will show later that the same trend is also valid for long-time dynamics.

Turning to examine the $\mathrm{BD}$ results, we find a significantly different behavior. While the experiments clearly show a marked slowing down of the short-time diffusion, the simulation data show a rather limited decrease of $D_{s}$. A plot of $D_{0} / D_{s}\left(\mathbf{q}_{c}\right)$ and $D_{0} / D_{s}\left(\mathbf{q}_{\mathrm{m}}\right)$, reported in Figure $6 \mathrm{~B}$ reveals that the numerical data initially follow the experimental ones quite closely and exhibit a similar concentration dependence at small $\phi$, but that they show a deviation from the experimental behavior for $\phi \gtrsim 0.15$. The dynamics on length scales of the monomer-monomer peak also do not exhibit a clear increase that could be fitted by a power-law, but their slowing down becomes more pronounced at high values of $\phi$, so that $D_{0} / D_{s}\left(\mathbf{q}_{\mathrm{m}}\right)$ becomes dominant and shows a larger increase with respect to $D_{0} / D_{s}\left(\mathbf{q}_{\mathrm{c}}\right)$. Hence, in contrast to the experiments where the arrest of the clusters drives the dynamical slowing down with the monomers being carried along with the clusters, in the simulations the reverse scenario occurs; particles tend to arrest by becoming trapped by their nearest neighbors and then drive a later arrest of the clusters themselves. We cannot determine the presence of an ideal glass transition with increasing $\phi$ in simulations, because the system becomes unstable (in the sense of a thermodynamic phase separation) for $\phi \geq 0.26$, a value surprisingly close to the experimentally observed glass transition,

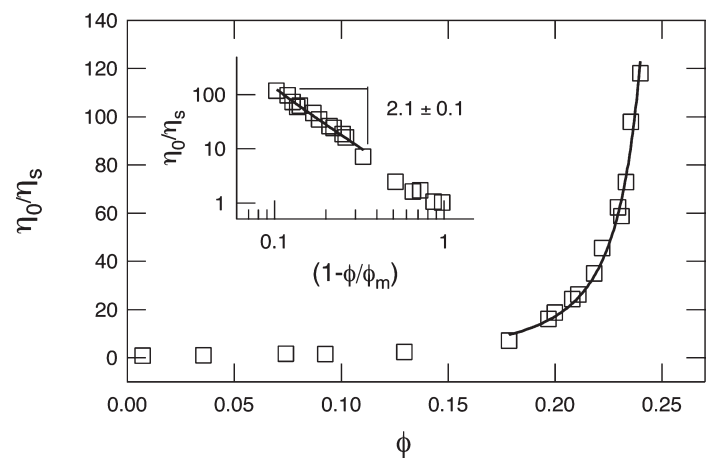

Figure 7. Volume fraction dependence of the reduced zero shear viscosity $\eta_{0} / \eta_{\mathrm{s}}$ at $T=5{ }^{\circ} \mathrm{C}$. The solid line is a fit to the data with a power law of the form $\eta_{\mathrm{o}} / \eta_{\mathrm{s}}=\left(1-\phi / \phi_{\mathrm{g}}\right)^{-\gamma}$ with $\phi_{\mathrm{g}}=0.267$ and $\gamma=2.1$ as illustrated in the inset.

although we cannot at present establish whether this is just a coincidence.

The discrepancy between experiments and simulations suggests that lysozyme clusters are in fact much less transient than predicted by our simple model. The deviations between $\mathrm{BD}$ and NSE data become clearly visible in the range between $\phi>0.074$, where the agreement is still good, and $\phi<0.169$, where the discrepancy is already large (see Figure 5C). In this regime, simulations indicate that the number of aggregates becomes significant and that the growth of the cluster size leads to static percolation for $\phi_{\mathrm{p}} \simeq 0.15$. ${ }^{23}$ We thus provide evidence that the dynamical arrest transition at $\phi_{\mathrm{g}}$ does not coincide with the static percolation transition at $\phi_{\mathrm{p}}$ but is situated at a larger value of $\phi$. However, the simplified effective potential that we are using to describe lysozyme solutions ceases to be valid for predicting the dynamics (but not the statics) for $\phi \gtrsim \phi_{\mathrm{p}}$, signaling that the emergence of a (transient) bonded network plays a role in the description of single-particle dynamics in terms of pairwise additive potential. The experimental results suggest the formation of long-lived transient clusters with lifetimes significantly longer than those observed in simulations. This is consistent with previous observations in cluster forming colloidal system, where particles were not observed to leave or join clusters within the observation time. ${ }^{3,36}$ It is quite conceivable that in this clusterdominated regime many-body interactions become important and result in a significantly enhanced stability of the clusters compared to those seen in the simulations. As a result, the cluster-driven dynamical arrest mechanism observed in experiments cannot be properly described by our simple model.

Long-Time Dynamics: Rheological Measurements and Mean-Squared Displacements. The limited time and q-dependence of the NSE data as well as the discrepancy between the experimental and simulation data clearly raises questions about the finite lifetime of the clusters and the existence of a "true" dynamical arrest transition, that is, the formation of a cluster-driven glass. To complement the short-time dynamics results, we therefore also study the macroscopic flow behavior of our lysozyme solutions. While NSE only probes short-time dynamics on length scales comparable to the cluster-cluster and monomer-monomer correlation lengths, with rheological measurements we are able to test whether the clusters are sufficiently long-lived to drive an arrest transition. Such a cluster-driven glass should occur at much smaller volume fractions than those where a corresponding monomeric fluid would exhibit a (repulsive or attractive) glass transition. ${ }^{37}$ 
The volume fraction dependence of the thus obtained estimate of the zero shear viscosity is shown in Figure 7, where $\eta_{0}$ has been normalized by the viscosity $\eta_{\mathrm{s}}$ of the pure solvent. A strong increase of the reduced viscosity is observed with increasing volume fraction. Moreover, this increase at sufficiently large $\phi$ is well captured by a power law of the form $\eta_{\mathrm{o}} / \eta_{\mathrm{s}} \sim\left(\phi_{\mathrm{g}}-\phi\right)^{-\gamma}$, which is shown in the inset. We find that the divergence volume fraction is $\phi_{\mathrm{g}} \simeq 0.267 \pm 0.005$, a value very close to that obtained previously from fitting the short-time diffusion coefficients, and $\gamma \simeq 2.1 \pm 0.1$.

This power-law behavior and related exponent are features that are commonly observed near an ideal glass transition, as predicted by $\mathrm{MCT}^{35}$ and also reported for colloidal glass-forming systems, such as hard spheres ${ }^{38-40}$ or colloids with short-range attractions. $^{41,42}$ In our fluid of clusters the volume fraction of arrest is much lower than the one observed in hard sphere systems $(\sim 0.58)$ and interestingly our results for the viscosity can be rescaled onto the hard sphere case using an effective volume fraction $\phi_{\mathrm{eff}}=\phi\left(\phi_{\mathrm{hs}} / \phi_{\mathrm{g}}\right) \approx \phi(2.22)$. This increase of the effective volume fraction provides further support for a long lifetime of the clusters and the existence of a cluster-driven dynamical arrest transition. Note that the exponents of 1.25 and 2.1 related to the divergence of the short-time dynamics and the viscosity are smaller than the one expected for hard spheres where the $\beta$ relaxation diverges with an eponent of 1.7 and the viscosity with 2.7 . A more accurate determination of the divergence parameters would be obtained by measurements closer to the transition, but unfortunately the procedure used to prepare samples does not allow us to reach volume fractions $\phi \gtrsim \phi_{\mathrm{g}}$.

From $\mathrm{BD}$ simulations, the calculation of the mean square displacement (MSD) $\left\langle r^{2}(t)\right\rangle$ offers a straightfoward way to evaluate the long-time self-diffusion coefficient $D_{\mathrm{L}}=\lim _{t \rightarrow \infty}\left\langle r^{2}\right.$ $(t)\rangle / 6$. Results for $D_{0} / D_{\mathrm{L}}$ are also reported in Figure 6B. Similarly to the case of short-time diffusion, simulations fail to predict a clear dynamic arrest in the studied $\phi$-window, displaying only a modest increase of $D_{0} / D_{\mathrm{L}}$ comparable to that of $D_{0} / D_{s}\left(\mathbf{q}_{\mathrm{m}}\right)$.

\section{DISCUSSION}

While the existence of a peak in $S_{\text {eff }}(\mathbf{q})$ that occurs at q-values below those predicted by $\mathbf{q}_{\mathrm{c}} \sim \phi^{1 / 3}$ does not yet allow to unambiguously conclude the existence of equilibrium clusters, a combination of SAXS/SANS and computer simulations has allowed us to verify that an essentially $\phi$-independent cluster peak position as it can be observed for weakly screened lysozyme solutions at lower temperatures and sufficiently high volume fractions is indeed the signature of the presence of finite-size clusters. Given the somewhat controversial state of the discussion on this subject, it is important to point out that cluster formation occurs as a result of a delicate balance of opposing forces, and minor changes in temperature or $\mathrm{pH}$ can shift this balance dramatically. When looking at recent studies, it is thus important to realize that the majority has been performed at higher temperatures only, where the balance is shifted toward Coulomb repulsion and cluster formation is limited. ${ }^{21,22}$ Moreover, when studies are not conducted at constant $\mathrm{pH}^{22}$ the resulting lower $\mathrm{pH}$ for higher concentrations increases the net charge of lysozyme, which also enhances Coulomb repulsion and reduces cluster formation.

The results shown in this manuscript not only provide additional support for the existence of equilibrium clusters in concentrated lysozyme solutions, ${ }^{3,21}$ but show that these clusters become the building blocks of an arrested state of lysozyme at $\phi \simeq 0.26$. Our experiments reveal a dramatic slowing down of the dynamics occurring both for short-time and long-time measurements. In particular, the study of the q-dependence of the intermediate scattering function using neutron spin echo measurements has allowed us to show that dynamical arrest is driven by the cluster mode, carrying with it an arrest also at the local level on NSE time scales. Moreover, rheological measurements have further supported the existence of equilibrium clusters with long lifetimes as the system undergoes also a macroscopic arrest in line with the arrested dynamics on local length scales.

Similarly to our observations, the NSE experiments of Porcar et al. ${ }^{21}$ have also shown evidence for the existence of equilibrium clusters. These authors also concluded that the clusters possess a lifetime that renders them stable over their experimental time window of less than $60 \mathrm{~ns}$. However, there are also differences due to different experimental conditions. This leads to a much weaker volume fraction dependence of the short time diffusion coefficient that they interpret as an indication of cluster growth only. When looking more carefully at the two studies, possible reasons for these differences become apparent. The experiments of Porcar et al. are conducted at lower $\mathrm{pH}$ values, where lysozyme carries a higher net charge and where cluster formation is thus less pronounced. Moreover, most of their measurements are conducted at a significantly higher temperature of $25^{\circ} \mathrm{C}$, which also favors monomers rather than cluster formation, ${ }^{3}$ and at lower overall volume fractions. In addition, the accessed experimental time window is significantly smaller, making it much more difficult to detect a deviation from single exponential decay of the intermediate scattering function and resulting in large error bars for measurements with slowly diffusing clusters. Finally, their analysis is based on an interpretation of the measured ISF that assumes basically monodisperse spherical clusters. Given all this, their findings actually do nicely demonstrate the existence of small dynamic clusters under conditions where larger electrostatic interactions limit cluster growth. This is also confirmed by the additional data presented very recently by these authors, where they also cover a larger time window. ${ }^{22}$ We now extend their observations to higher volume fractions and conditions where cluster growth is much more pronounced and also provide evidence for the emergence of a dynamical arrest transition.

Additional insights into the transient nature of the clusters is also gained from BD simulations based on a simple effective lysozyme-lysozyme potential, composed of a short-range attraction plus a long-range repulsion, which maintains pairwise additivity and spherical symmetry. The agreement between simulations and experiments on the dynamics is remarkable at low $\phi$, while a dramatic discrepancy occurs as soon as $\phi$ grows, in proximity to the static percolation transition. The failure of $\mathrm{BD}$ simulations to correctly reproduce the experimental data paradoxically reinforces the idea of cluster-dominated arrest; indeed, while in simulations the clusters remain always transient and monomer exchange is very frequent between clusters (as indicated by the stronger decay of the short-time diffusion on the monomer peak scale) at the studied $T$, experiments clearly show that this is not the case and that clusters become long-lived (as also observed for colloids ${ }^{3,36}$ ) up to the point where they form a stress-bearing arrested structure.

The failure of the numerical calculations in describing the true nature of the clusters can be attributed to different factors. First of all, the neglection of hydrodynamic effects ${ }^{33,34}$ when clusters 
grow in size and number could be an important source of discrepancy. However, recent studies of aggregation have revealed that the role of hydrodynamic interactions is limited in three-dimensional simulations. ${ }^{43}$ Moreover, a comparison between $\mathrm{BD}$ and a novel algorithm developed to incorporate hydrodynamic interactions (fast lubrication dynamics, FLD) for colloidal suspensions interacting via short-range attraction and long-range repulsion at comparable volume fractions has demonstrated remarkable agreement for both the microstructure and dynamics. ${ }^{44}$ This suggests that the structural and dynamic properties of these types of systems are not very sensitive to the effects of hydrodynamic interactions. Nevertheless, the influence of hydrodynamic interactions on systems comparable with ours still warrants further studies. There are results from very recent numerical simulations investigating phase separation and arrest in suspensions of purely attractive particles interacting with a relatively long-range attraction using the fluid particle dynamics (FPD) method ${ }^{45}$ that contradict the conclusions in ref 44 and indicate that hydrodynamic interactions may indeed play an important role in the gelation of colloids. It would thus be important to extend these investigations to the case of mixed potentials where clusters form.

Furthermore, we believe that our lysozyme-lysozyme effective potential misses some important ingredients that can be relevant close to the arrest transition. The hydrophobic nature of the short-range attraction certainly calls for a more appropriate patchy description. ${ }^{46-48}$ In addition, the monomer-monomer effective description may start to fail when multiple bonds are involved and the system is organized into the preferred cluster structures. Indeed, when the system is organized into large structures, either many-body terms in the form of an additional repulsive barrier may be acting, or particles may ultimately fall in a deep van-der-Waals minimum that we have not considered in our simplified treatment. Moreover, while we have assumed that the protein charge remains constant irrespective of whether the proteins are present as monomers or in clusters, this may not be the case and the protein charge in the clusters could be lower. In all three cases, the bonds within the clusters would be much more persistent in time. Certainly these discrepancies become clearly visible close to the percolation transition when the system forms a macroscopic cluster, however we do not have sufficient data to judge the exact location in $\phi$ where clusters become dominant. It would be interesting to understand whether there is a welldefined point where this happens (e.g., close to percolation) or whether a smooth crossover takes place. In this respect, our simulation results for $D_{0} / D_{s}\left(\mathbf{q}_{\mathrm{c}}\right)$ and $D_{0} / D_{\mathrm{s}}\left(\mathbf{q}_{\mathrm{m}}\right)$ show a clear intersection (Figure $6 \mathrm{~B}$ ) between the two sets of data very close to the percolation transition.

The existence of a percolation transition at around $\phi_{\mathrm{p}} \sim 0.15$ is important for addressing the nature of the observed clusterdriven dynamical arrest. Is it a Wigner glass of clusters ${ }^{2,49}$ or more an attractive glass (or gel) of clusters $?^{50}$ The fact that arrest takes place at $\phi_{\mathrm{g}} \gg \phi_{\mathrm{p}}$ strongly indicates that the transition is driven by attractive bonds. However, there could be regimes, outside the current experimental window (lower $T$, lower $\mathrm{pH}$ ), where a Wigner glass of lysozyme clusters similar to the recently reported cluster glass found in highly deionized clay suspensions ${ }^{8,51}$ could exist. While we currently have no conclusive information on the exact nature of the cluster solid that forms at $\phi \geq \phi_{\mathrm{g}}$ we comment that we now have a clear manifestation of the transient nature of the bonds, despite the presence of the long-range repulsive barrier, which indeed provides a possible mechanism for an

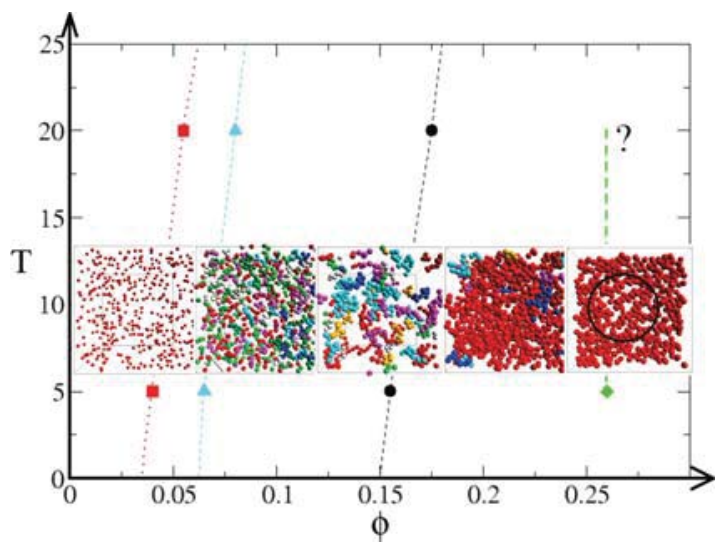

Figure 8. Schematic state diagram of lysozyme solutions with representative snapshots taken from simulations at fixed $T=5^{\circ} \mathrm{C}$. Particles are colored according to their clusters. For small $\phi$ only selected clusters are shown, while for large $\phi$ only partial slabs of full configurations are shown to avoid overcrowding and to show the gel interconnection. From left to right, we observe monomers $(\phi=0.037)$, clusters which with increasing $\phi$ change shape from spherical $(\phi=0.074)$ to more elongated $(\phi=0.125)$, transient percolation (red aggregate, $\phi=0.169$ ) and finally an arrested state (indicative snapshot taken from simulations at $\phi=0.25$ ). The lines from left to right indicate the monomer-to-cluster transition, defined in two ways: the locus when the average weight cluster size exceeds 2 (squares) and that where $\mathbf{q}_{\mathrm{c}}$ becomes constant in $S(\mathbf{q})$ (triangles), the percolation transition (circles) and the arrest transition (diamonds).

attractive glass transition of clusters well above the establishment of a percolation network. This reinforces the original interpretation ${ }^{3,17}$ that there is a wide regime of concentration where the system exists in the form of truly equilibrium clusters.

Finally, it is interesting to comment on another arrest mechanism found for lysozyme solutions in the presence of salt. ${ }^{52}$ Here the formation of an arrested spinodal network below the metastable liquid-gas coexistence curve, similar to experimental observations for short-ranged attractive colloids, ${ }^{53,54}$ has been recently demonstrated. These different routes to arrest result in totally different nonergodic systems. ${ }^{50}$ The arrest transition in the cluster fluid at low ionic strength is characterized by a homogeneous cluster fluid that exhibits structure only on relatively short length scales given by the cluster-cluster and monomer-monomer correlation length. Moreover, its low-q structure factor manifests the interplay of repulsive and attractive interactions in the form of a strongly decreased $S(0)$ followed by the cluster peak. Quite in contrast, the arrested spinodal network shows the typical large correlation length of a bicontinuous system created by spinodal decomposition process frozen in time as a result of the dynamic arrest of the dense phase. This arrested spinodal decomposition manifests itself also by a very much enhanced forward scattering and the formation of a typical spinodal peak. ${ }^{55-57}$

\section{SUMMARY AND CONCLUSIONS}

In this work, we have provided a full characterization of the structural and dynamic properties of salt-free lysozyme solutions. Using a number of complementary tools, such as SAXS, NSE, rheology, as well as numerical simulations, we have been able to demonstrate the existence of long-lived equilibrium clusters at low enough temperatures and high enough volume fractions.

The evolution of the cluster formation with volume fraction and temperature and the occurrence of an arrest transition at 
high enough volume fractions are shown in the schematic state diagram reported in Figure 8. The transition from a monomer to a cluster-dominated fluid has been estimated from an inspection of the effective structure factors, both from experiments and theory, as well as from the cluster distributions. Snapshots taken from the simulations at $T=5{ }^{\circ} \mathrm{C}$ are shown in Figure 8 to illustrate the evolution of the clusters with increasing volume fractions. For very low $\phi \lesssim 0.05$, only monomers and few small clusters are found. At higher volume fractions, transient equilibrium clusters dominate and the position of the cluster peak in $S_{\text {eff }}$ now remains essentially constant. The clusters possess a broad size distribution and their shape becomes more elongated as $\phi$ increases, similarly to observations made with cluster-forming colloidal suspensions with competing interactions. ${ }^{4,36}$

The combination of BD simulations and NSE measurements has provided us with new insight into the lifetime of the equilibrium clusters formed under these conditions. While the experimentally determined dynamic structure factors closely agree with the simulations at low and intermediate concentrations, strongly supporting the transient nature of the clusters, this changes dramatically in the region $\phi \sim 0.10-0.15$. Indeed, for $\phi>0.15$ we observe large discrepancies between experimental and numerical results. Not only do we see a much more pronounced slowing down for the cluster mobility, but the dynamic structure factor also becomes almost independent of $\mathbf{q}$ for $\mathbf{q}>\mathbf{q}_{\mathbf{c}}$, indicating that the clusters have a much longer lifetime than predicted by our model.

As demonstrated in Figures 6 and 7, our experiments also indicate the existence of an arrest transition for $\phi \gtrsim 0.26$ that not only occurs on the short time scales probed by NSE but is also confirmed at the macroscopic level as seen by rheological measurements. It is the long-lived nature of the clusters that is at the origin of the simultaneous dynamical arrest at all length scales.

It is interesting to note that the arrest transition does not coincide with the appearance of geometric percolation at $\phi \sim 0.15$, where the formation of a transient network is observed in the simulations. We may however speculate that the percolation threshold could be related to the onset of major discrepancies between $\mathrm{BD}$ and experiments. The fact that $S_{\text {eff }}$ continues to show a cluster peak even well inside the percolating regime is a signature of the fact that the clusters are the building blocks of the network. This is also supported by the dominance of the divergence in $D_{s}(\mathbf{q})$ at the cluster peak, quite in contrast to what is seen in the simulations.

Our findings unambiguously demonstrate that the peculiar structural properties found in salt-free lysozyme solutions at high volume fractions are not only the result of typical density fluctuations in attractive monomeric fluids but caused by true equilibrium clusters. However, there still remain a number of open questions that warrant further investigations. In particular, the exact nature of the arrest transition found in this cluster fluid and its connection to the liquid-solid transition through an arrested spinodal decomposition observed at higher salt concentrations, where electrostatic repulsions are effectively screened, and the repulsive glass transition that we expect to find at higher temperatures where repulsions dominate and cluster formation is suppressed, remains to be further investigated.

\section{ASSOCIATED CONTENT}

S Supporting Information. We provide a detailed account of the new SAXS measurements used to generate Figure 1 and provide additional information on the determination of the viscosity values reported in Figure 7.

\section{AUTHOR INFORMATION}

\section{Corresponding Author}

*E-mail: (E.Z.) emanuela.zaccarelli@phys.uniroma1.it; (A.S.) Anna.Stradner@unifr.ch.

\section{ACKNOWLEDGMENT}

We thank C. De Michele for providing the code to generate the snapshots of Figures 2C and 8. We acknowledge support from the Swiss National Science Foundation, the Adolphe Merkle Foundation, the EU NoE "SoftComp" NMP3-CT2004-502235 and the Marie Curie Network on Dynamical Arrest of Soft Matter and Colloids MRTNCT-2003-504712. E.Z. and F. S. thank ERC-226207-PATCHYCOLLOIDS and ITN-234810COMPLOIDS for support.

\section{REFERENCES}

(1) Groenewold, J.; Kegel, W. J. Phys. Chem. B 2001, 105, 11702-11709.

(2) Sciortino, F.; Mossa, S.; Zaccarelli, E.; Tartaglia, P. Phys. Rev. Lett. 2004, 93, 055701.

(3) Stradner, A.; Sedgwick, H.; Cardinaux, F.; Poon, W. C. K.; Egelhaaf, S. U.; Schurtenberger, P. Nature 2004, 432, 492-495.

(4) Campbell, A. I.; Anderson, V. J.; van Duijneveldt, J.; Bartlett, P. Phys. Rev. Lett. 2005, 94, 208301.

(5) Sedgwick, H.; Kroy, K; Salonen, A.; Robertson, M. B.; Egelhaaf, S. U.; Poon, W. C. K. Eur. Phys. J. E 2005, 16, 77-80.

(6) Dibble, C. J.; Kogan, M.; Solomon, M. J. Phys. Rev. E 2006, $74,041403$.

(7) Gopalakrishnan, V.; Zukoski, C. F. Phys. Rev.E 2007, 75, 021406.

(8) Shalkevich, A.; Stradner, A.; Bhat, S. K.; Muller, F.; Schurtenberger, P. Langmuir 2007, 23, 3570.

(9) Zhang, F.; Skoda, M. W. A.; Jacobs, R. M. J.; Martin, R. A.; Martin, C. M.; Schreiber, F. J. Phys. Chem. B 2007, 111, 251-259.

(10) Pan, W.; Galkin, O.; Filobelo, L.; Nagel, R. L.; Vekilov, P. G. Biophys. J. 2007, 92, 267-277.

(11) Gliko, O.; Pan, W.; Katsonis, P.; Neumaier, N.; Galkin, O.; Weinkauf, S.; Vekilov, P. G. J. Phys. Chem. B 2007, 111, 3106-3114.

(12) Boutet, S.; Robinson, I. K. Phys. Rev. E 2007, 75, 021913.

(13) Destainville, N. Phys. Rev. E 2008, 77, 011905.

(14) Javid, N.; Vogtt, K.; Krywka, C.; Tolan, M.; Winter, R. Phys. Rev. Lett. 2007, 99, 028101.

(15) Nicolai, T.; Durand, D. Curr. Opin. Colloid Interface Sci. 2007, $12,23-28$.

(16) Ianeselli, L.; Zhang, F.; Skoda, M. W. A.; Jacobs, R. M. J.; Martin, R. A.; Callow, S.; Prevost, S.; Schreiber, F. J. Phys. Chem. B 2010, 114, 3776-3783.

(17) Stradner, A.; Cardinaux, F.; Schurtenberger, P. J. Phys. Chem. B 2006, 110, 21222-21231.

(18) Shukla, A.; Mylonas, E.; Di Cola, E.; Finet, S.; Timmins, P.; Narayanan, T.; Svergun, D. I. Proc. Natl. Acad. Sci. U.S.A. 2008, 105, 5075-5080.

(19) Stradner, A.; Cardinaux, F.; Egelhaaf, S. U.; Schurtenberger, P. Proc. Natl. Acad. Sci. U.S.A. 2008, 105, E75-E75.

(20) Shukla, A.; Mylonas, E.; Di Cola, E.; Finet, S.; Timmins, P.; Narayanan, T.; Svergun, D. I. Proc. Natl. Acad. Sci. U.S.A. 2008, 105, E76-E76.

(21) Porcar, L.; Falus, P.; Chen, W.-R; Faraone, A.; Fratini, E.; Hong, K.; Baglioni, P.; Liu, Y. J. Phys. Chem. Lett. 2010, 1, 126-129.

(22) Liu, Y.; Porcar, L.; Chen, J.; Chen, W.-R.; Falus, P.; Faraone, A.; Fratini, E.; Hong, K.; Baglioni, P. J. Phys. Chem. B. 2010, in press, DOI:10.1021/jp109333c. 
(23) Cardinaux, F.; Stradner, A.; Schurtenberger, P.; Sciortino, F.; Zaccarelli, E. Europhys. Lett. 2007, 77, 48804.

(24) Schleger, P.; Alefeld, B.; Barthelemy, J. F.; Ehlers, G.; Farago, B.; Giraud, P.; Hayes, C.; Kollmar, A.; Lartigue, C.; Mezei, F.; Richter, D. Physica B 1997, 241, 164-165.

(25) Farago, B. Physica B 1999, 268, 270-276.

(26) Belloni, L. J. Phys.: Condens. Matter 2000, 12, 549.

(27) Belloni, L. J. Chem. Phys. 1986, 85, 519-526.

(28) Koppel, D. E. J. Chem. Phys. 1972, 57, 4814.

(29) Pusey, P. N.; Tough, R. J. A. In Dynamic Light Scattering: Applications of Photon Correlation Spectroscopy; Pecora, R., Ed.; Plenum: New York, 1985.

(30) Broccio, M.; Costa, D.; Liu, Y.; Chen, S.-H. J. Chem. Phys. 2006, 124,084501

(31) Beenakker, C. W. J.; Mazur, P. Physica A 1984, 126, 349-370.

(32) Egelhaaf, S.; Lobaskin, V.; Bauer, H.; Merkle, H.; Schurtenberger, P. Eur. Phys. J. E 2004, 13, 153164.

(33) Banchio, A. J.; Gapinski, J.; Patkowski, A.; Häussler, W.; Fluerasu, A.; Sacanna, S.; Holmqvist, P.; Meier, G.; Lettinga, M. P.; Nägele, G. Phys. Rev. Lett. 2006, 96, 138303.

(34) Banchio, A. J.; McPhie, M. G.; Nägele, G. J. Phys.: Condens. Matter 2008, 20, 4213.

(35) Götze, W. In Liquids, Freezing and the Glass Transition; Hansen, J. P., Levesque, D., Zinn-Justin, J., Eds.; North-Holland: Amsterdam, 1991; pp 287-503.

(36) Sedgwick, H.; Egelhaaf, S. U.; Poon, W. C. K. J. Phys.: Condens. Matter 2004, 16, 4913.

(37) Sciortino, F. Nat. Mater. 2002, 1, 145-146.

(38) Segré, P. N.; Meeker, S. P.; Pusey, P. N.; Poon, W. C. K. Phys. Rev. Lett. 1995, 75, 958.

(39) Phan, S.-E.; Russel, W. B.; Cheng, Z.; Zhu, J.; Chaikin, P. M.; Dunsmuir, J. H.; Ottewill, R. H. Phys. Rev. E 1996, 54, 6633-6645.

(40) Meeker, S. P.; Poon, W. C. K.; Pusey, P. N. Phys. Rev. E 1997, $55,5718-5722$.

(41) Mallamace, F.; Tartaglia, P.; Chen, W. R.; Faraone, A.; Chen, S.-H. J. Phys.: Condens. Matter 2004, 16, 4975.

(42) Puertas, A. M.; Zaccarelli, E.; Sciortino, F. J. Phys.: Condens. Matter 2005, 17, L271-L277.

(43) Yamamoto, R.; Kim, K.; Nakayama, Y.; Miyazaki, K.; Reichman, D. R. J. Phys. Soc. Jpn. 2008, 77, 084804.

(44) Bybee, M. D. Ph.D. Thesis, University of Illinois at UrbanaChampaign (2009).

(45) Furukawa, A.; Tanaka, H. Phys. Rev. Lett. 2010, 104, 245702.

(46) Lomakin, A.; Asherie, N.; Benedek, G. B. Proc. Nat. Ac. Sci. 1999, 96, 9465-9468.

(47) Liu, H.; Kumar, S. K; Sciortino, F. J. Chem. Phys. 2007, 127, 084902.

(48) Gogelein, C.; Nagele, G.; Tuinier, R.; Gibaud, T.; Stradner, A.; Schurtenberger, P. J. Chem. Phys. 2008, 129, 085102.

(49) Toledano, J. C. F.; Sciortino, F.; Zaccarelli, E. Soft Matter 2009, $5,2390-2398$.

(50) Zaccarelli, E. J. Phys.: Condens. Matter 2007, 19, 323101.

(51) Ruzicka, B.; Zulian, L.; Zaccarelli, E.; Angelini, R.; Sztucki, M.; Moussad, A.; Ruocco, G. Phys. Rev. Lett. 2010, 104, 085701.

(52) Cardinaux, F.; Gibaud, T.; Stradner, A.; Schurtenberger, P. Phys. Rev. Lett. 2007, 99, 118301.

(53) Buzzaccaro, S.; Rusconi, R.; Piazza, R. Phys. Rev. Lett. 2007, 99, 098301.

(54) Lu, P. J.; Zaccarelli, E.; Ciulla, F.; Schofield, A. B.; Sciortino, F.; Weitz, D. A. Nature 2008, 453, 499-503.

(55) Zaccarelli, E.; Lu, P. J.; Ciulla, F.; Weitz, D. A.; Sciortino, F. J. Phys.: Condens. Matter 2008, 20, 494242.

(56) Gibaud, T.; Schurtenberger, P. J. Phys.: Condens. Matter 2009, $21,322201$.

(57) Gibaud, T.; Cardinaux, F.; Bergenholtz, J.; Stradner, A.; Schurtenberger, P. Soft Matter 2011, 7, 857. 